\title{
Primates en las colecciones biológicas de Antioquia: estado y potencial de investigación
}

\author{
Primates in the biological collections of Antioquia: Status and research potential
}

\author{
Sebastián García-R $\mathbf{R}^{1,2,3 *}$
}

\begin{abstract}
Resumen
En Colombia se reconoce la existencia de 38 especies de primates, de las cuales 13 se distribuyen en Antioquia. Sin embargo, la mayoría de las investigaciones se enfocan en aspectos ecológicos mientras que son escasos los estudios sobre distribución y taxonomía. Para evaluar la representatividad de primates se consultaron tres colecciones biológicas locales, dos nacionales y cinco internacionales. Se registran diez especies en las colecciones del departamento, siendo Alouatta seniculus la más representada y faltando únicamente A. palliata, Aotus zonalis y Ateles fusciceps. Por su parte, las colecciones nacionales y del exterior albergan ejemplares de nueve especies complementando las colecciones locales con registros de $A$. fusciceps. Se resalta la escasez de información del origen de especímenes en algunas colecciones, la necesidad de actualización en identificaciones taxonómicas y la importancia de generar estrategias que aporten al ingreso de material biológico a las colecciones.
\end{abstract}

Palabras claves: Colección Teriológica Universidad de Antioquia, mamíferos, Museo La Salle, Museo Universitario Universidad de Antioquia

\begin{abstract}
There are 38 recognized primate species in Colombia, including 13 that occur in Antioquia. Most of the researches are focused in ecology while studies on distribution and taxonomy are scarce. Three local biological collections were consulted, two nationals and five internationals in order to evaluate the primates representativeness. Ten species are reported in the department collections, with Alouatta seniculus being the most represented and missing only A. palliata, Aotus zonalis and Ateles fusciceps. National and international collections house specimens of nine species complementing the local collections with records of $A$. fusciceps. The scarcity of information on the origin of specimens in some collections is highlighted, as well as the need of updating in taxonomic identifications and the importance of generating strategies that contribute to the income of biological material into the collections.
\end{abstract}

Keywords: Colección Teriológica Universidad de Antioquia, mammals, La Salle Museum, University Museum Universidad de Antioquia

\footnotetext{
1. Fundación Proyecto Primates. Bogotá D.C., Colombia

2. Grupo Mastozoología, Universidad de Antioquia, Medellín, Colombia

3. Departamento de Ciencias Biológicas, Universidad de los Andes, Bogotá D.C., Colombia

* Autor de correspondencia: <sebasgr93@gmail.com>
} 


\section{INTRODUCCIÓN}

Antioquia es un departamento rico en fauna y con amplia variedad de zonas de vida a causa de su ubicación entre las cordilleras Occidental y Central, y por su posición en la esquina noroccidental de Colombia donde inicia el denominado puente centroamericano, zona de alto significado zoogeográfico (Cuartas-Calle y Muñoz-Arango, 2003). En Antioquia se registran 13 especies de primates: Alouatta palliata (Gray, 1849), Alouatta seniculus (Linnaeus, 1766), Aotus griseimembra Elliot, 1912, Aotus lemurinus (I. Geoffroy, 1843), Aotus zonalis Goldman, 1914, Ateles fusciceps Gray, 1866, Ateles hybridus (I. Geoffroy, 1829), Cebus capucinus (Linnaeus, 1758), Cebus versicolor Pucheran, 1845, Lagothrix lagothricha (Humboldt, 1812), Saguinus geoffroyi (Pucheran, 1845), Saguinus leucopus (Günther, 1876) y Saguinus oedipus (Linnaeus, 1758) (García-R et al, 2018). Este valor representa el $34,2 \%$ del total de especies reconocido para Colombia $(\mathrm{n}=38)$ (Asociación Primatológica Colombiana, 2020a; Ramírez-Chaves, Suárez-Castro, y González-Maya, 2016).

Las investigaciones con primates en Antioquia se han concentrado en temas relacionados con ecología seguidos por estudios genéticos o moleculares, donde algunos corresponden a análisis que incluyen muestras de diferentes localidades del departamento (García-R et al, 2018). Por su parte, los estudios sobre distribución, taxonomía y sistemática son exiguos, lo que concuerda con el panorama nacional (Stevenson, Pérez-Torres, y Muñoz-Saba, 2006; Stevenson, Guzmán, y Defler, 2010; García-R et al, 2018). En este aspecto, las colecciones biológicas cumplen un papel fundamental al ser archivos de la presencia de organismos en un lugar y tiempo determinado. Allí, los especímenes constituyen la mayor fuente de información acerca de la distribución geográfica de un animal o planta (Simmons y MuñozSaba, 2005).

El Museo de Ciencias Naturales de La Salle (Museo CNS), fundado en 1913 por los Hermanos de La Salle, se dedica a la preservación, investigación y divulgación del patrimonio cultural y natural de Antioquia. Los Hermanos de La Salle documentaron la biota antioqueña aportando su conocimiento en campos específicos de la ornitología, botánica, mastozoología y otras ramas de las ciencias naturales. Sus colecciones son custodiadas por el Instituto Tecnológico Metropolitano de Medellín desde el año 2006 (Museo CNS, 2018). Por su parte, la colección de Ciencias Naturales del Museo Universitario de la Universidad de Antioquia (MUUA) fue fundada en 1942 como "Museo de Ciencias Naturales de la Universidad de Antioquia" e incluía colecciones de minerales, esqueletos, fósiles y algunos animales naturalizados. En 1970 pasó a ser colección del actual Museo Universitario al igual que el Museo de Antropología. Cuenta con 18.000 ejemplares entre animales naturalizados, esqueletos, pieles de estudio, minerales, fósiles e ilustraciones científicas (MUUA, 2020). Finalmente, la Colección Teriológica de la Universidad de Antioquia, que hace parte del Grupo de Mastozoología, fue establecida formalmente en enero de 2003 como parte del Instituto de Biología de la Universidad de Antioquia. Proviene, en su mayoría, de las colecciones realizadas por el profesor Javier Muñoz-Arango entre 1980 y 1995; posteriormente, la colección ha sido nutrida a partir de investigaciones en las Cordillera Central y Occidental de Colombia (Sergio Solari, Prof. Asoc., Inst. de Biología, Universidad de Antioquia, 2018, comunicación personal).

Para esta contribución se consultaron las colecciones biológicas de Antioquia, que albergan especímenes del orden Primates, con el objetivo de evaluar su estado y potencial de investigación en lo concerniente a primates no humanos. Adicionalmente, se incluyen algunos comentarios acerca de la representatividad de este grupo en colecciones nacionales e internacionales para resaltar el esfuerzo de muestreo en el departamento.

\section{MATERIALES Y MÉTODOS}

La evaluación del estado de las colecciones biológicas del departamento se realizó a partir de la revisión directa del material depositado en la Colección Teriológica de Antioquia (CTUA) y en la colección de Mastozoología del Museo Universitario de la Universidad de Antioquia (MUA-MAM). Por su parte, el catálogo del Museo de Ciencias Naturales de La Salle, Instituto Tecnológico Metropolitano (CSJ-m) 
fue consultado de manera virtual. No se incluyó información de la colección biológica de la Universidad EAFIT dado que no cuenta con especímenes de primates; por su parte, aunque la colección biológica de la Universidad CES alberga restos de algunos individuos no fue posible acceder a su información.

Adicionalmente, se accedió a la información de la colección de mamíferos del Instituto de Ciencias Naturales (ICN) de la Universidad Nacional de Colombia a través del recurso biovirtual.unal.edu.co, y a la colección de mamíferos del Instituto de Investigación de Recursos Biológicos Alexander von Humboldt (IAvH) a través de la plataforma del SIB Colombia. Cabe aclarar que no se obtuvo información de registros de primates de Antioquia en el ICN a través del SIB Colombia. Por su parte, a través del portal Vernet (http://portal.vertnet.org), se accedió a los catálogos de las colecciones del exterior que cuentan con especímenes provenientes de Antioquia, incluyendo: American Museum of Natural History (AMNH), Field Museum of Natural History (FMNH), Museum of Comparative Zoology, Harvard University (MCZ), Royal Ontario Museum (ROM) y National Museum of Natural History - Smithsonian Institution (USNM).

Toda la información recolectada fue contrastada con listados nacionales de los últimos diez años (APC, 2020a; Defler, 2010; Ramírez-Chaves et al, 2016; Solari et al, 2013); se corrigió la taxonomía de los especímenes con identificaciones desactualizadas de acuerdo con la Guía de Cambios Taxonómicos (versión 4.0) (APC, 2020b).

\section{RESULTADOS}

Las colecciones biológicas del departamento de Antioquia albergan 88 especímenes de primates no humanos $(\mathrm{CTUA}=15, \mathrm{MUA}-\mathrm{MAM}=59, \mathrm{CSJ}-\mathrm{m}=14)$ agrupados en siete familias, once géneros y 22 especies. Dos de las especies corresponden a "monos del Viejo Mundo", Macaca sp. y Pan troglodytes Blumenbach, 1799, dispuestos en la sección de exhibición del MUA-MAM (tabla 1). De las 20 especies de primates neotropicales registradas, cinco son endémicas de Colombia (APC 2020a) y once se encuentran bajo alguna categoría de amenaza según la Unión Internacional para la Conservación de la Naturaleza (Carretero, Guzmán-Caro, Palacios y Stevenson, 2020; Carretero, Guzmán-Caro y Stevenson, 2020; de la Torre, Morales, Link, Palacios y Stevenson 2015; Link, Mittermeier y Urbani, 2019; Link et al, 2019; Link, Urbani, Stevenson y Mittermeier, 2020; Méndez-Carvajal, Link, Mittermeier y Palacios, 2019; Morales-Jiménez y de la Torre, 2008; Morales-Jiménez, Link y Stevenson, 2008; Rodríguez et al, 2020; Stevenson, Link y Guzman-Caro, 2020) (tabla 1).

La información disponible indica que las recolectas se realizaron entre 1913 y 1979 en la CSJ-m; 2014 en la CTUA y entre 1976 y 2005 en el MUAMAM. La mayoría de los especímenes carecen de datos de localidad; el 40,9\% no tiene información del departamento (CTUA=5, MUA-MAM=31), mientras que $13,6 \%($ MUA-MAM=12) corresponden a animales aportados por el Parque Zoológico Santa Fe de Medellín, cuya localidad original se desconoce. Los demás especímenes $(\mathrm{n}=40)$ provienen de ocho departamentos de Colombia, siendo la mayoría de Antioquia y Córdoba (tabla 1) y corresponden a diez de las 13 especies que existen en Antioquia, donde $A$. seniculus es la más representada mientras que $A$. palliata, A. fusciceps y $A$. zonalis no cuentan con registros.

En cuanto a su identidad taxonómica, los 88 especímenes están identificados a nivel de familia, cinco hasta género y 82 hasta especie. De estos, se requiere la corrección en la identificación de 14 especímenes con base en su distribución y en recientes hipótesis taxonómicas (tabla 2) (Boubli, Rylands, Farias, Alfaro, y Lynch-Alfaro, 2012; Buckner, Lynch-Alfaro, Rylands, y Alfaro, 2015; Byrne et al, 2016; Defler, 2010; Link et al, 2015; Lynch-Alfaro et al, 2015; RuizGarcía et al, 2010).

Por último, en colecciones por fuera del departamento y de Colombia, se encontraron 81 registros de especímenes de primates de Antioquia correspondientes a nueve especies, siendo $A$. seniculus la más representada y sumando diez registros de A. fusciceps (tabla 3), especie que no está representada en las colecciones locales revisadas. Los años y colectores de los especímenes, según la información disponible, 
Tabla 1. Especímenes de primates no humanos depositados en las colecciones biológicas de Antioquia.

\begin{tabular}{|c|c|c|c|c|c|}
\hline Familia & Especie & Especímenes $\ln (r$ & Colección & Departamento & UICN \\
\hline \multirow{6}{*}{ Aotidae } & Aotus brumbacki ${ }^{A}$ & 1.0 & MUA-MAM & Meta & VU \\
\hline & Aotus griseimembra & 2.0 & CSJ-m & Tolima & VU \\
\hline & Aotus lemurinus & 3.0 & CSJ-m & Antioquia & VU \\
\hline & Aotus sp. & 2.0 & MUA-MAM & & - \\
\hline & Aotus trivirgatus & 3.0 & MUA-MAM & & LC \\
\hline & Aotus vociferans & 2.0 & CTUA; MUA-MAM & Vaupés & LC \\
\hline \multirow{4}{*}{ Atelidae } & Alouatta seniculus & 24.0 & CSJ-m; CTUA; MUA-MAM & $\begin{array}{l}\text { Antioquia, Cesar, } \\
\text { Córdoba, NA, Zoo }\end{array}$ & LC \\
\hline & Ateles belzebuth & 1.0 & CTUA & & EN \\
\hline & Ateles hybridus & 4.0 & CSJ-m; CTUA; MUA-MAM & Antioquia, NA & CR \\
\hline & Lagothrix lagothricha ${ }^{\mathrm{B}}$ & 6.0 & CSJ-m; MUA-MAM & Antioquia, NA, Zoo & $\mathrm{CR}^{\mathrm{C}}$ \\
\hline \multirow{7}{*}{ Callitrichidae } & Cebuella pygmaea & 1.0 & MUA-MAM & & LC \\
\hline & Leontocebus fuscus & 2.0 & MUA-MAM & NA, Vaupés & LC \\
\hline & Saguinus geoffroyi & 3.0 & MUA-MAM & Chocó, Żoo & NT \\
\hline & Saguinus inustus & 1.0 & CTUA & Vaupés & LC \\
\hline & Saguinus leucopus ${ }^{A}$ & 12.0 & CTUA; MUA-MAM & Antioquia, NA & EN \\
\hline & Saguinus oedipus ${ }^{\mathrm{A}}$ & 4.0 & MUA-MAM & Córdoba, NA, Zoo & CR \\
\hline & Saguinus sp. & 1.0 & MUA-MAM & & - \\
\hline \multirow{6}{*}{ Cebidae } & Cebus albifrons & 1.0 & MUA-MAM & \multirow[b]{2}{*}{$\begin{array}{l}\text { Antioquia, Córdoba, } \\
\text { Valle del Cauca, NA }\end{array}$} & LC \\
\hline & Cebus capucinus & 4.0 & CSJ-m; CTUA; MUA-MAM & & LC \\
\hline & Cebus sp. & 1.0 & MUA-MAM & & - \\
\hline & Cebus versicolor $^{\mathrm{A}}$ & 2.0 & CSJ-m; CTUA & Antioquia & EN \\
\hline & Saimiri cassiquiarensis & 2.0 & MUA-MAM & Zoo & LC \\
\hline & Sin identificar & 1.0 & MUA-MAM & & - \\
\hline Cercopithecidae & Macaca sp. & 1.0 & MUA-MAM & & - \\
\hline Hominidae & Pan troglodytes & 3.0 & MUA-MAM & NA, Zoo & EN \\
\hline Pitheciidae & Plecturocebus ornatus $^{A}$ & 1.0 & CSJ-m & Meta & VU \\
\hline
\end{tabular}

Categorías de la Unión Internacional para la Conservación de la Naturaleza; NA: Espécimen sin información de localidad;

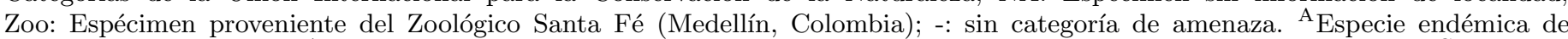
Colombia (APC, 2020a). ${ }^{\text {A }}$ Lagothrix lagothricha lugens (CSJ-m 0362), Subespecie endémica de Colombia (APC, 2020a). ${ }^{\mathrm{C}}$ Categoría correspondiente a Lagothrix lagothricha lugens.

Tabla 2. Correcciones sugeridas a especímenes depositados en las colecciones de Antioquia.

\begin{tabular}{|c|c|c|}
\hline Especie* $^{*}$ & Código & Observación \\
\hline \multirow{2}{*}{ Aotus griseimembra } & CSJ-m 0360 & Antes Aotus sp. Procedente de El Espinal, Tolima \\
\hline & CSJ-m 0603 & Antes Aotus trivirgatus. Procedente de El Espinal, Tolima \\
\hline Aotus vociferans & CTUA - & Antes Aotus sp. Procedente de Vaupés \\
\hline Ateles hybridus & CSJ-m 0380 & Antes Ateles sp. Procedente de Remedios, Antioquia \\
\hline Cebuella pygmaea & MUA-MAM 285 & Antes Callithrix pygmea \\
\hline Cebus capucinus & CSJ-m 0358 & Antes Cebus hipoleucus. Procedente de Palmira, Valle del Cauca \\
\hline \multirow{2}{*}{ Cebus versicolor } & CTUA 2343 & Antes Cebus albifrons. Procedente de Segovia, Antioquia \\
\hline & CSJ-m 0359 & Antes Cebus albifrons. Procedente de San Luis, Antioquia \\
\hline \multirow{2}{*}{ Leontocebus fuscus } & MUA-MAM 283 & Antes Saguinus mystax. Procedente de Vaupés \\
\hline & MUA-MAM 284 & Antes Saguinus mystax \\
\hline Plecturocebus ornatus & CSJ-m 0361 & Antes Callicebus sp. Procedente de Villavicencio, Meta \\
\hline Saguinus inustus & CTUA - & Antes Saguinus sp. Procedente de Vaupés \\
\hline Saimiri cassiquiarensis & $\begin{array}{l}\text { MUA-MAM 460; } \\
\text { MUA-MAM 461 }\end{array}$ & Antes Saimiri sciurus \\
\hline
\end{tabular}

*Identificación ajustada por el autor. 
Tabla 3. Especímenes de primates no humanos depositados en colecciones biológicas por fuera del departamento.

\begin{tabular}{l|l|c}
\hline Especie & Colección (Número de especímenes) & Total especímenes \\
\hline Alouatta seniculus & AMNH (5), FMNH (1), ICN(3), IAvH (5), ROM (1), USNM (4) & 19 \\
\hline Aotus griseimembra & & 9 \\
\hline Aotus lemurinus & AMNH (4), FMNH (2), IAvH (3) & 8 \\
\hline Ateles fusciceps** & AMNH (3), FMNH (5) & 10 \\
\hline Cebus albifrons*** & FMNH (2), IAvH (5), USNM (3) & 2 \\
\hline Cebus capucinus & IAvH (2) & 3 \\
\hline Lagothrix lagothricha**** & (3) & 1 \\
\hline Saguinus leucopus & IAvH (3) & 17 \\
\hline Saguinus oedipus & IAvH (1) & 12 \\
\hline
\end{tabular}

AMNH: American Museum of Natural History; FMNH: Field Museum of Natural History; IAvH: Instituto Alexander von Humboldt; ICN: Instituto de Ciencias Naturales; MCZ: Museum of Comparative Zoology, Harvard University; ROM: Royal Ontario Museum; USNM: National Museum of Natural History, Smithsonian Institution

*Identificados en catálogos como Aotus lemurinus lemurinus: AMNH 37805, 37813, 37814, 37815; y FMNH 69612, 69613. Identificados en catálogo como Aotus lemurinus griseimembra: IAvH-M 1513, 1514, 2865. Corregidos considerando su información de distribución y elevación (Defler, 2010).

**Identificados en catálogo como Ateles geoffroyi: IAvH-M 2680, 2683, 2686, 2687, 2870. Corregidos de acuerdo con la Guía de cambios taxonómicos de la APC (2020b).

*** Decomisados. Provenientes de Medellín: IAvH-M 2860, 2866.

****Decomisado. Proveniente de Medellín. IAvH 6122.

son: AMNH entre 1913 y 1915 (L.E. Miller, C. Chapman, H.S. Boyle, y H.A. Ward); FMNH en 1950 (P. Hershkovitz); ICN entre 1964 y 1981 (Ernesto Barriga Bonilla), IAvH entre 1971 y 1974 (José Rodríguez, N. Peterson, Ernesto Barriga Bonilla, Socorro Sierra y Horacio Echeverri), MCZ en 1834 (Henry Augustus Ward); ROM en 1950 (sin información del colector) y USNM entre 1970 y 1972 (N. Peterson).

\section{DISCUSIÓN}

A pesar de que Antioquia es un departamento rico en fauna, ubicado en un hotspot de biodiversidad (Ceballos y Ehrlich, 2006; Loyola, Kubota, da Fonseca, y Lewinsohn, 2009), y con representación de especies de primates endémicas y amenazadas, carece de investigaciones en temas relacionados con taxonomía y sistemática (García-R et al, 2018). Las colecciones biológicas, como archivos o bibliotecas de la biodiversidad de una región, son una herramienta fundamental para evaluar patrones biogeográficos, cambios en patrones de distribución y realizar estudios comparativos, entre otros (Garbino y Martins-Junior, 2018; Serrano-Villavicencio, Vendramel y Garbino, 2017). Los especímenes, en muchos casos, son los únicos testigos de la fauna que habitó un lugar determinado en un momento de la historia, por lo que su preservación y la calidad de la información asociada, son tareas primordiales.

Los especímenes de primates no humanos depositados en las colecciones biológicas de Antioquia provienen de ocho departamentos de Colombia. Allí están representadas 20 especies de primates neotropicales, que corresponden al 52,6\% de las reconocidas para Colombia (APC, 2020a; Ramírez-Chaves et al, 2016). Por su parte, se encuentran representadas diez de las 13 especies que existen en Antioquia, donde únicamente A. palliata, A. fusciceps y A. zonalis carecen de registros en las colecciones locales. Estas especies, además, se incluyen entre las menos estudiadas en el departamento por lo que resulta necesario generar propuestas de investigación que aborden aspectos de su historia natural y genética, entre otros (García-R et al, 2018).

En cuanto al estado de las colecciones, se encontró que una parte importante de los especímenes carecen de información de localidad, principalmente en el MUA-MAM, ya sea por la ausencia de datos del lugar de origen o porque corresponden a especímenes en cautiverio. La mayoría de los especímenes están identificados a nivel de especie, aunque 14 requieren ajustes de acuerdo con recientes consideraciones taxonómicas (CTUA=3, MUA-MAM=5, CSJ-m=6). 
Estas imprecisiones pueden asociarse con la antigüedad de los registros. Por ejemplo, los especímenes del CSJ-m fueron recolectados hace más de 40 años por Pedro González (1913) y los hermanos Lasallistas Nicéforo María (1918-1937), Marco Antonio Serna (1966-1979) y José Ignacio Isaza (1967-1979). La misma situación se observa en las colecciones nacionales y del exterior, donde las últimas recolectas sistemáticas de primates corresponden a las décadas de 1970 y 1980. Estas colecciones albergan especímenes de nueve especies del departamento de Antioquia complementando las colecciones locales con ejemplares de A. fusciceps; siendo fuentes de información importantes al momento de realizar estudios sobre distribución de esta especie en Colombia.

La generación de estrategias que aporten al ingreso de material biológico a las colecciones ayudaría a la obtención de nuevos registros en la región incrementando el esfuerzo de muestreo y aportando a futuros estudios en taxonomía y sistemática a nivel nacional. Algunas alternativas comprenden la creación de convenios con Centros de Atención y Valoración de fauna silvestre (CAVs), zoológicos u otras instituciones o la recolección de heces y el almacenamiento de registros acústicos, siempre con detallada información asociada.

\section{AGRADECIMIENTOS}

A los curadores de las colecciones biológicas consultadas por facilitar el acceso a los especímenes y la información asociada. Al Museo de Ciencias Naturales de La Salle, un proyecto cultural del ITM por proveer la información de catálogo y a los evaluadores por sus aportes al mejoramiento del manuscrito.

\section{CONFLICTO DE INTERESES}

El autor declara no tener conflictos de intereses.

\section{REFERENCIAS}

American Museum of Natural History. (2020, abril 12) AMNH Mammal Collections. Subset of data for VERTNET. Recuperado de: http://ipt.vertnet.org:8080/ipt/resource. do? $\mathrm{r}=$ amnh $\_$mammals
Asociación Primatológica Colombiana. (2020a, agosto 13) Primates de Colombia. Recuperado de: https://drive. google.com/file/d/1LTbK-F4V5lGh1gJe3iMHvZqs3dlk $\mathrm{rid} / \mathrm{view}$

Asociación Primatológica Colombiana. (2020b, agosto 13). Especies de primates colombianos. Guía de cambios taxonómicos (versión 3.0). Recuperado de: https://www. asoprimatologicacolombiana.org/uploads/1/1/4/7/ 11474090/guía_cambios_taxonómicos_primates_4.0.pdf

Boubli, J.P., Rylands, A.B., Farias, I.P., Alfaro, M.E., y LynchAlfaro, J. (2012). Cebus phylogenetic relationships: A preliminary reassessment of the diversity of the untufted capuchin monkeys. American Journal of Primatology, 74(4), 381-393. doi:10.1002/ajp.21998

Buckner, J.C., Lynch-Alfaro, J., Rylands, A.B., y Alfaro, M.E. (2015). Biogeography of the marmosets and tamarins (Callitrichidae). Molecular Phylogenetics and Evolution, 82, 413-425. doi:10.1016/j.ympev.2014.04.031

Byrne, H., Rylands, A.B., Carneiro, J.C., Lynch-Alfaro, J.W., Bertuol, F., Da Silva, M.N., Messias, M., Groves, C.P., Mittermeier, R.A., Farias, I., Hrbek, T., Scheneider, H. Sampaio, I., y Boubli, J.P. (2016). Phylogenetic relationships of the New World titi monkeys (Callicebus): first appraisal of taxonomy based on molecular evidence. Frontiers in Zoology, 13(1), 10. doi:10.1186/s12983-016$0142-4$

Carretero, X., Guzmán-Caro, D., Palacios, E., y Stevenson, P.R. (2020). Plecturocebus ornatus. The IUCN Red List of Threatened Species 2020: e.T39928A17974735. doi:10.2305/IUCN.UK.20202.RLTS.T39928A17974735.en

Carretero, X., Guzmán-Caro, D., y Stevenson, P.R. (2020). Aotus brumbacki. The IUCN Red List of Threatened Species 2020: e.T39915A17923405. doi:10.2305/IUCN.UK.20202.RLTS.T39915A17923405.en

Cuartas-Calle, C.A., y Muñoz-Arango, J. (2003). Lista de los mamíferos (Mammalia: Theria) del departamento de Antioquia, Colombia. Biota Colombiana, 4 (1), 65-78. http://revistas.humboldt.org.co/index.php/ biota/article/view/124/123

Ceballos, G., y Ehrlich, P.R. (2006). Global mammal distributions, biodiversity hotspots, and conservation. Proceedings of the National Academy of Sciences, 103(51), 19374-19379. doi:10.1073/pnas.0609334103

Defler, T.R. (2010). Historia natural de los primates colombianos. Bogotá D.C, Colombia: Universidad Nacional de Colombia

de la Torre, S., Morales, A. L., Link, A., Palacios, E., y Stevenson, P. (2015). Cebus versicolor. The IUCN Red List of Threatened Species 2015: e.T39952A81281674. doi:10.2305/IUCN.UK.2015.RLTS.T39952A81281674.en

Field Museum of Natural History. (2020, abril 12). Field Museum of Natural History (Zoology) Mammal Collection. Subset of data for VERTNET. Recuperado de: http://fmipt.fieldmuseum.org:8080/ipt/resource.do? $\mathrm{r}=$ fmnh_mammals

Garbino, G.S., y Martins-Junior, A.M. (2018). Phenotypic evolution in marmoset and tamarin monkeys (Cebidae, Callitrichinae) and a revised genus-level classification. Molecular Phylogenetics and Evolution, 118, 156-171. 
doi:10.1016/j.ympev.2017.10.002

García-R, S., Montilla, S.O., Bustamante-Manrique, V., Bustamante-Manrique, S., Cepeda-Duque, C., SánchezLondoño, J.D., y Ramírez-Chaves, H.E. (2018). Estado de la investigación primatológica en el eje cafetero y Antioquia, Colombia. Neotropical Primates, 24 (2), 56-63. http://www.primate-sg.org/neotropical-primates-242/

Instituto de Ciencias Naturales, Facultad de Ciencias, Universidad Nacional de Colombia. (2020, marzo 26). Colecciones en Línea. Recuperado de: http://www.biovirtual.unal. edu.co

Instituto de Investigación de Recursos Biológicos Alexander von Humboldt (2019). Colección de Mamíferos del Instituto de Investigación de Recursos Biológicos Alexander von Humboldt (IAvH-M). 9446 registros. doi:10.15472/aghjkw

Link, A., Valencia, L.M., Céspedes, L.N., Duque, L.D., Cadena, C.D., y Di Fiore, A. (2015). Phylogeography of the critically endangered brown spider monkey (Ateles hybridus): Testing the riverine barrier hypothesis. International Journal of Primatology, 36(3), 530-547. doi:10.1007/s10764-015-9840-6

Link, A., Mittermeier, R.A., y Urbani, B. (2019). Aotus griseimembra. The IUCN Red List of Threatened Species 2019: e.T1807A17922228. doi:10.2305/IUCN.UK.20192.RLTS.T1807A17922228.en

Link, A., Muniz, C., Rylands, A.B., Mourthé, Í., Cornejo, F.M., Urbani, B., Mittermeier, R.A., Stevenson, P.R., Palacios, E., Boubli, J., Shanee, S., de la Torre, S., y Moscoso, P. (2019). Ateles belzebuth. The IUCN Red List of Threatened Species 2019: e.T2276A17928557. doi:10.2305/IUCN.UK.20192.RLTS.T2276A17928557.en

Link, A., Urbani, B., Stevenson, P.R., y Mittermeier, R.A. (2020). Ateles hybridus. The IUCN Red List of Threatened Species 2020: e.T39961A17929680. dpo:10.2305/IUCN.UK.20202.RLTS.T39961A17929680.en

Loyola, R.D., Kubota, U., da Fonseca, G.A., y Lewinsohn, T.M. (2009). Key Neotropical ecoregions for conservation of terrestrial vertebrates. Biodiversity and Conservation, 18, 2017-2031. doi:10.1007/s10531-008-9570-6

Lynch-Alfaro, J.W., Boubli, J.P., Paim, F.P., Ribas, C.C., da Silva, M.N.F., Messias, M.R., Rohe, F., Mercês, M.P., Silva Junior, J.S., Silva, C.R., Pinho, G.M., Koshkarian, G., Nguyen, M.T.T., Harada, M.L., Rabelo, R.M., Queiroz, H.L., Alfaro, M.E., y Farias, I.P. (2015). Biogeography of squirrel monkeys (genus Saimiri): South-central Amazon origin and rapid pan-Amazonian diversification of a lowland primate. Molecular Phylogenetics and Evolution, 82, 436-454. doi:10.1016/j.ympev.2014.09.004

Méndez-Carvajal, P., Link, A., Mittermeier, R.A., y Palacios, E. (2019). Saguinus geoffroyi. The IUCN Red List of Threatened Species 2019: e.T41522A17932085. doi:10.2305/IUCN.UK.20193.RLTS.T41522A17932085.en

Morales-Jiménez, A.L., y de la Torre, S. (2008). Aotus lemurinus. The IUCN Red List of Threatened Species 2008: e.T1808A7651803. doi:10.2305/IUCN.UK.2008.RLTS.T1808A7651803.en

Morales-Jiménez, A.L., Link, A., y Stevenson, P.
(2008). Saguinus leucopus. The IUCN Red List of Threatened Species 2008: e.T19819A9019454. doi:10.2305/IUCN.UK.2008.RLTS.T19819A9019454.en

Museo de Ciencias Naturales de La Salle (Museo CNS). (2018). Historia. Recuperado de https://museo.itm.edu. co/pages/historia.html

Museo Universitario de la Universidad de Antioquia (MUUA). (2020). Acerca del Museo. Recuperado de https://bit.ly/ 3eSWWRS

Harvard University Museum of Comparative Zoology. (2013). Museum of Comparative Zoology, Harvard University, Subset of data for VERTNET. doi:hrefhttp://doi.org/10.15468/p5rupv10.15468/p5rupv

Orrell, T. (2020, abril 12): NMNH Extant Specimen Records. v1.34. National Museum of Natural History, Smithsonian Institution. Dataset/Occurrence. Recuperado de: https://collections.nmnh.si.edu/ipt/resource?r= nmnh_extant_dwc-a\&v=1.34

Ramírez-Chaves, H.E., Suárez-Castro, A.F., y GonzálezMaya, J.F. (2016). Cambios recientes a la lista de los mamíferos de Colombia. Mammalogy Notes, 3(1), 1-9. https://mammalogynotes.org/ojs/index.php/ $\mathrm{mn} /$ article/view/60/47

Rodríguez, V., Defler, T.R., Guzmán-Caro, D., Link, A., Mittermeier, R.A., Palacios, E., y Stevenson, P.R. (2020). Saguinus oedipus. The IUCN Red List of Threatened Species 2020: e.T19823A115573819. doi:10.2305/IUCN.UK.20202.RLTS.T19823A115573819.en

Royal Ontario Museum. (2020, abril 12). Mammalogy Collection - Royal Ontario Museum. Subset of data for VERTNET. http://gbif.rom.on.ca:8180/ipt/ resource.do? $\mathrm{r}=$ mamm

Ruiz-García, M., Castillo, M.I., Vásquez, C., Rodríguez, K., Pinedo-Castro, M., Shostell, J., y Leguizamon, N. (2010). Molecular phylogenetics and phylogeography of the white-fronted capuchin (Cebus albifrons; Cebidae, Primates) by means of mtCOII gene sequences. Molecular Phylogenetics and Evolution, 57(3), 1049-1061. doi:10.1016/j.ympev.2010.09.002

Serrano-Villavicencio, J.E., Vendramel, R.L., y Garbino, G.S.T. (2017). Species, subspecies, or color morphs? Reconsidering the taxonomy of Callicebus Thomas, 1903 in the PurusMadeira interfluvium. Primates, 58(1), 159167. doi:10.1007/s10329-016-0555-x

Simmons, J.E., y Muñoz-Saba, Y. (Eds.). (2005). Cuidado, manejo y conservación de las colecciones biológicas. Bogotá D.C, Colombia: Universidad Nacional de Colombia. http://www.ibiologia.unam.mx/pdf/ directorio/c/cervantes/clases/sistem/Cuidado_Manejo_ y_Conservacion_de_las_Colecciones_Biologicas.pdf

Solari, S., Munoz-Saba, Y., Rodríguez-Mahecha, J.V., Defler, T.R., Ramírez-Chaves, H.E. y Trujillo, F. (2013). Riqueza, endemismo y conservación de los mamíferos de Colombia. Mastozoología. Neotropical, 20(2), 301-365. https://mn.sarem.org.ar/article/riqueza-endemismo-yconservacion-de-los-mamiferos-de-colombia/

Stevenson, P.R., Pérez-Torres, J., y Muñoz-Saba, Y. (2006). Estado del conocimiento sobre los mamíferos terrestres y voladores en Colombia. Tomo II. En: M.E Chaves y M. 
Santamaría (Eds.). Informe sobre el avance en el conocimiento y la información de la biodiversidad 19982004 (pp. 151-170). Bogotá, Colombia: Instituto de Investigación de Recursos Biológicos Alexander von Humboldt.

Stevenson, P.R., Guzmán, D.C., y Defler, T.R. (2010). Conservation of Colombian primates: an analysis of published research. Tropical Conservation Science, 3(1), 45-62. doi:10.1177/194008291000300105

Stevenson, P.R., Link, A., y Guzman-Caro, D. (2020). Lagothrix lagothricha ssp. lugens. The IUCN Red List of Threatened Species 2020: e.T39926A17927955. doi:10.2305/IUCN.UK.20202.RLTS.T39926A17927955.en 